Dhaka Univ. J. Biol. Sci. 19(2): 137-144, 2010 (July)

\title{
REDUCED SUSCEPTIBILITY TO FLUROQUINOLONE AND MOLECULAR ANALYSIS OF CIPROFLOXACINE RESISTANCE IN SALMONELLA TYPHI IN BANGLADESH
}

\author{
A. B. M. Sarwer-E-Alom Sarker, SAmir K. SaHA ${ }^{1}$, \\ MAKSUDA ISLAM AND M. A. HoSSAIN* \\ Department of Biochemistry and Molecular Biology, University of Dhaka, \\ Dhaka-1000, Bangladesh
}

Key words: Reduced susceptibility, Typhoid fever, Salmonella typhi, Fluoroquinolone

\begin{abstract}
The study of the trend of infection, susceptibility to antibiotics and molecular level analysis of the cause of reduced susceptibility of Salmonella typhi isolates from the patients in Bangladesh were studied. Out of 9040 blood cultures obtained during the study period, 1266 (14.0\%) showed significant growth. Nearly three-fourths of the positive blood cultures were due to S. typhi and rests were mostly of $S$. paratyphi A. The prevalence was highest between the age group 25 and 60 months. Male showed slightly higher rate of infection than female. Among all $943 \mathrm{~S}$. typhi isolates, 42.6, 42 and $41.4 \%$ were sensitive to ampicillin, cotrimoxazol and chloramphenicol, respectively. All isolates were sensitive to ceftriaxon and ceftazidim; 9 isolates were ciprofloxacin resistant, others were moderate to highly sensitive; whereas, only $2.2 \%$ isolates were sensitive and almost all (97.8\%) were found resistant to nalidixic acid. The Estrip test among 411 isolates showed the MIC value of 53 isolates nearer to the very sensitive $(<0.125 \mu \mathrm{g} / \mathrm{ml}), 252$ isolates between 0.125 and $0.5 \mu \mathrm{g} / \mathrm{ml}, 95$ isolates between 0.5 and $2.0 \mu \mathrm{g} / \mathrm{ml}$ and rest other 11 isolates showed from > 2.0 $\mu \mathrm{g} / \mathrm{ml}$ to very highly resistant $(512 \mu \mathrm{g} / \mathrm{ml})$. VNTR pattern of all ciprofloxacine resistant $S$. typhi was also same. Restriction fragment analysis of gyrase- $A$ gene indicated point mutations in different loci that bear the cause of being resistant to ciprofloxacin.
\end{abstract}

\section{Introduction}

Typhoid fever is endemic in Bangladesh as so in the Indian subcontinent with high prevalence among under-five children.(1) Prevalence of multi-drug resistant typhoid strains lead to use the ciprofloxacin as the drug of choice for routine outpatient treatment. This resulted in rapid emergence of Salmonella enterica serovar typhi isolates with reduced susceptibility to ciprofloxacin and associated delay in response or treatment failure to ciprofloxacin therapy. These strains are still considered 'susceptible' to ciprofloxacin based on the National Committee for Clinical Laboratory Standards (NCCLS) cut-off values [i.e., zone $\leq 20 \mathrm{~mm}$; minimum inhibitory concentration (MIC) $\geq$ $2.0 \mu \mathrm{g} / \mathrm{ml}$, and are termed as nalidixic acid-resistant S. typhi (NARST) on the basis of

${ }^{*}$ Corresponding author. ${ }^{1 D}$ Department of Microbiology, Dhaka Shishu (Children) Hospital, Dhaka-1207, Bangladesh. 
their resistance to nalidixic acid. At present, based on NCCLS guidelines, S. typhi strains with true resistance to ciprofloxacin, (MIC $\geq 2.0 \mu \mathrm{g} / \mathrm{ml}$ ) have been identified; the highest MIC level reported from clinical isolate of $S$. typhi is $>32 \mu \mathrm{g} / \mathrm{ml}$. The decreased level of susceptibility of NARST strains to ciprofloxacin arisen from point mutations in the QRDR region of $g y r A$ gene, either at ser- 83 or asp- 87 or both.(2) Present work was undertaken to study the prevalence of reduced susceptibility to fluroquinolone and then molecular analysis of ciprofloxacine resistant Salmonella serover tphi in Bangladesh.

\section{Materials and Methods}

The blood samples were collected from unvaccinated patients who were clinically diagnosed with typhoid fever and admitted to Dhaka Shishu hospital, Popular Diagnostic Center and Kumudiny Hospital. Although, sample collection was started from 1997, samples collected from June, 2005 to August, 2006 were used. Patients' age, sex, duration of typhoid fever, name of the Salmonella typhi isolates, antibiotic sensitivity and minimum inhibitory concentration (MIC) for fluoroquinolone (ciprofloxacin) were recorded. Blood cultures were made using standard methodology on Blood-Agar, Chocolate-Agar and MacConkey-Agar medium. The isolates of $S$. typhi were confirmed by standard biochemical (API-20E system, BioMeriux, France) and serologic (Murex Diagnostics, Dartford, England) means. The antibiotic susceptibility test was done by Disc Diffusion method, using Muller-Hinton agar plate(3) according to the NCCLS guidelines. ${ }^{(4)}$ All statistical analyses were done using EpiData software. DNA was extracted from pure culture of Salmonella typhi on MacConkey-agar plate by heat-lysis method. Molecular typing was done by multiplex PCR for Variable-Number Tandem Repeats (VNTR) using primers flanking three VNTR loci (TR1, TR2 and TR3) (Table 1) as described by Liu et al. The PCR amplified product of gyraseA gene was confirmed by running it through $1 \%$ polyaccrylamide gel and after cutting into fragments by Hinf-1 restriction enzyme. The probability of point mutation was confirmed by running it through $2 \%$ polyaccrylamide gel.

\section{Results and Discussion}

Out of the 9040 blood cultures obtained during the study period, $1266(14.0 \%)$ yielded significant growth. Nearly three-fourths of the positive blood cultures $(74.5 \%$, 943 of 1266) were $S$. typhi and others were mostly S. paratyphi A. The isolation rate from boys and girls was apparently similar (Fig. 1).

The present laboratory-based study, showed the prevalence of $S$. typhi among the age group between 2 and 10 years.

Moreover, the high rate of isolation and degree of bacteremia in younger children suggest that they are more vulnerable to the infection. This argues against the view that 
typhoid fever typically is mild and-or sub-clinical in younger patients and mandates a reassessment of the epidemiology and severity of typhoid fever in the community in endemic areas. The passive nature of case detection in the present study however may have biased the data toward more severe disease because only very ill patients typically are taken to physician and furthermore only a portion of them considered being able to afford the investigation cost may have been sent to the referral Centre. Despite this limitation the large number of patients evaluated in this study that were confirmed to

\section{Total : Male : Female}

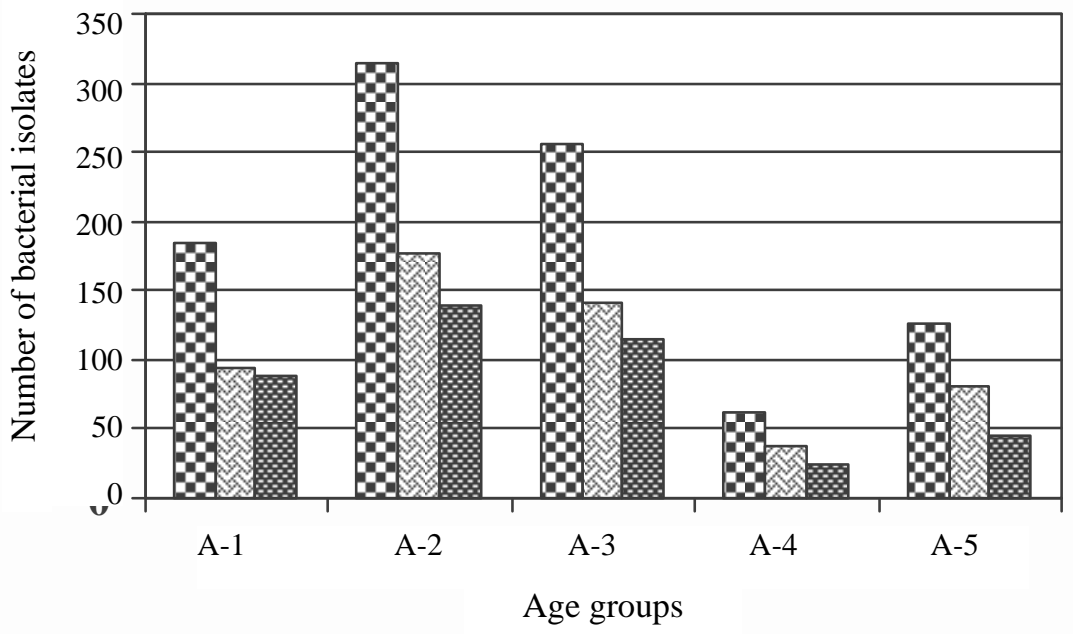

Fig. 1. Distribution of bacterial isolates with respect to different age groups of the patients (A1 is for 0 to 24 months; A2 for 25 to 60 months; A3 for 61 to 120 months; A4 for 121 to 180 months and A5 for 181 months to maximum age of our sample collections.)

have typhoid fever proved the prevalence of the disease. Moreover, the patients undertaken to this study were largely from the middle and upper income population of Dhaka city who have access to pipe water. Thus the incidence of typhoid fever in urban slum and rural areas of Bangladesh, which do not have safe water supplies, might be even higher than present data would indicate. The isolation rate was highest between the age group 25 and 60 months and male showed slightly higher rate of infection than female. These results will help in taking decision for efficacious trial of typhoid vaccine within this age group, particularly pre-school children.

In addition, the isolation rate was highest in the summer and monsoon season (May to October), with peaks in September (9.7\% in 2005 and 12.3\% in 2006) and was relatively low from November to March (Fig. 2). This trend was also observed in the year preceding to the study period. Among all 943 Salmonella typhi isolates studied, $42.6 \%$ of the total isolates were sensitive to ampicillin, whereas $57.4 \%$ resistant; $42 \%$ of the total 
isolates were sensitive to cotrimoxazol, whereas $57.4 \%$ are resistant. On the other hand, all isolates were sensitive to ceftriaxon and ceftazidim; $41.4 \%$ of the total isolates were sensitive to chloramphenicol and $58.6 \%$ are resistant; 9 isolates collected were ciprofloxacin resistant, others were moderate to highly sensitive; however, only $2.2 \%$ isolates were nalidixic acid sensitive and almost all (97.8\%) were found resistant (Fig. 3).

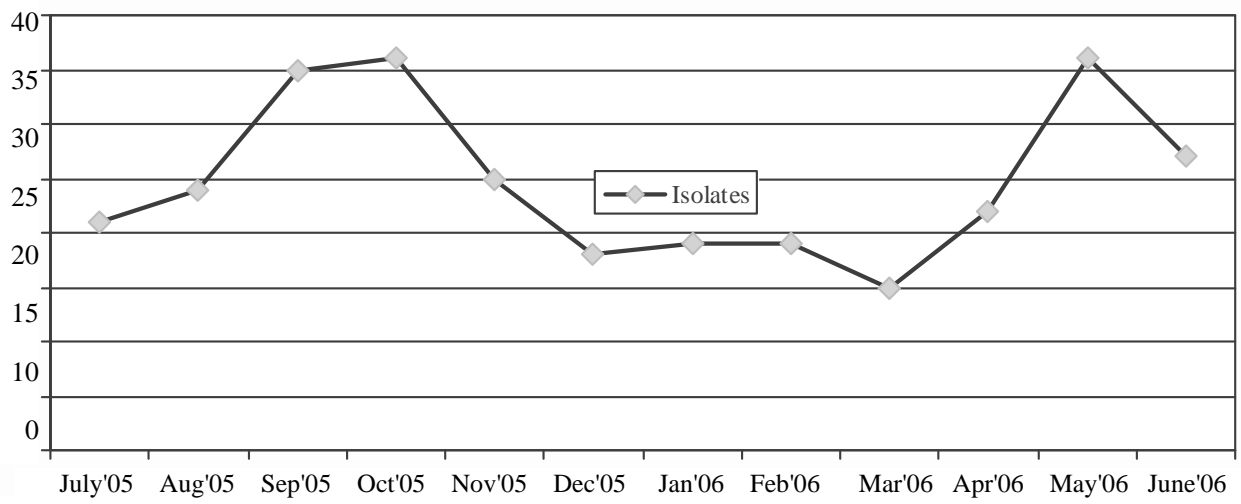

Fig. 2. Incidence of $S$. typhi isolates with patients during a year.

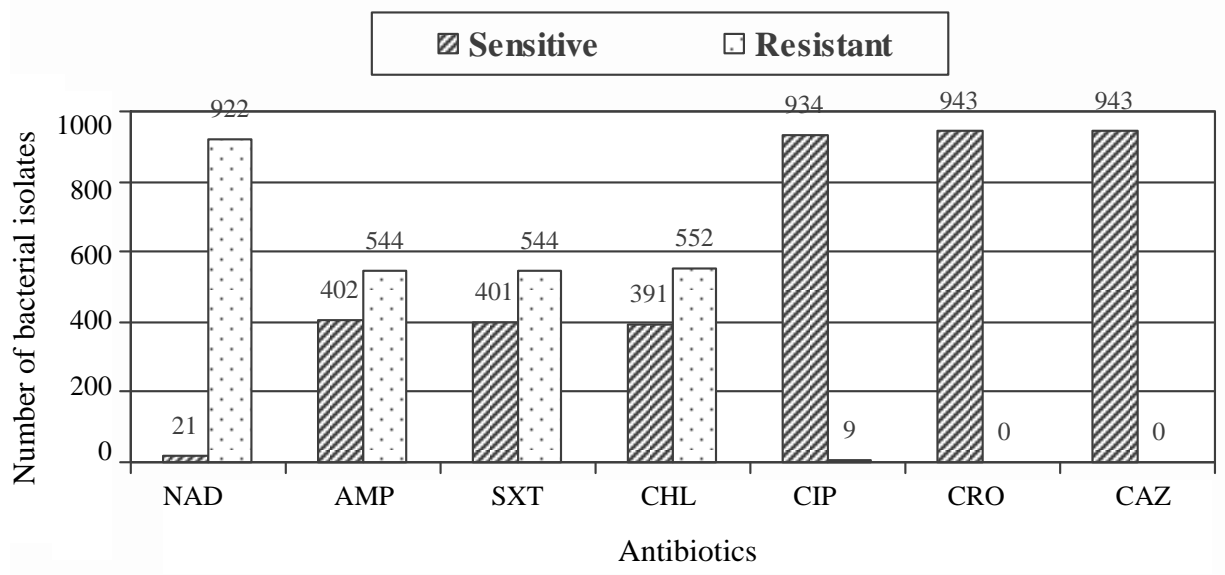

Fig. 3. Antibiogram of Salmonella typhi to different antibiotics.

Present study shows a drastic increase in the annual proportion of reduced fluoroquinolone susceptibility from 3.9 to $23.5 \%$ among all the Salmonella serover typhi in Bangladesh between 1999 and January 2007. Among all 943 S. typhi isolates studied, E-strip test was performed for 411 isolates of which 53 showed the MIC value nearer to the very sensitive $(<0.125 \mu \mathrm{g} / \mathrm{ml}), 252$ showed between 0.125 to $0.5 \mu \mathrm{g} / \mathrm{ml}$, 95 showed between 0.5 to $2.0 \mu \mathrm{g} / \mathrm{ml}$ and rest other 11 isolates showed from $>2.0 \mu \mathrm{g} / \mathrm{ml}$ to vary highly resistant e.g. $512 \mu \mathrm{g} / \mathrm{ml}$ (Fig. 4). 


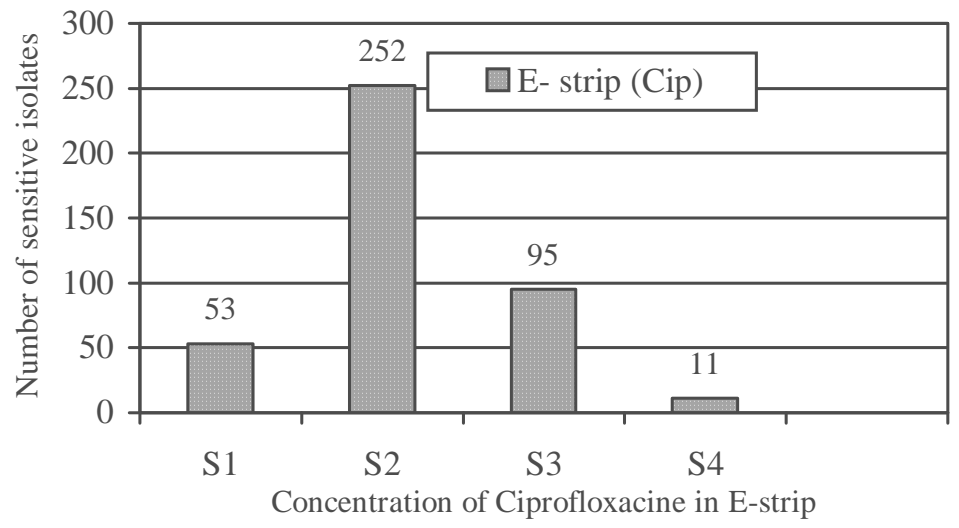

Fig. 4. Status of susceptibility of $S$. typhi isolates to Fluoroquinolone. ( $\mathrm{S} 1$ is $<0.125$, S2 is in between 0.125 and $0.5, \mathrm{~S} 3$ is in between 0.5 and 2.0 and $\mathrm{S} 4$ is $>2.0 \mu \mathrm{g} / \mathrm{ml}$, respectively.)

All the ciprofloxacine resistant isolates were also highly resistant to ampicillin $(>256$ $\mu \mathrm{g} / \mathrm{ml})$, cotrimoxazole $(>32 \mu \mathrm{g} / \mathrm{ml})$, chloramphenicol $(>256 \mu \mathrm{g} / \mathrm{ml})$, ciprofloxacin $(>32$ $\mu \mathrm{g} / \mathrm{ml})$ and nalidixic acid $(>256 \mu \mathrm{g} / \mathrm{ml})$, and were susceptible to ceftriaxone $(0.094 \mu \mathrm{g} / \mathrm{ml})$ according to their MIC of respective antibiotics. All three isolates were found to be identical by Api 20E score (4404552) and VNTR type (Fig. 5).

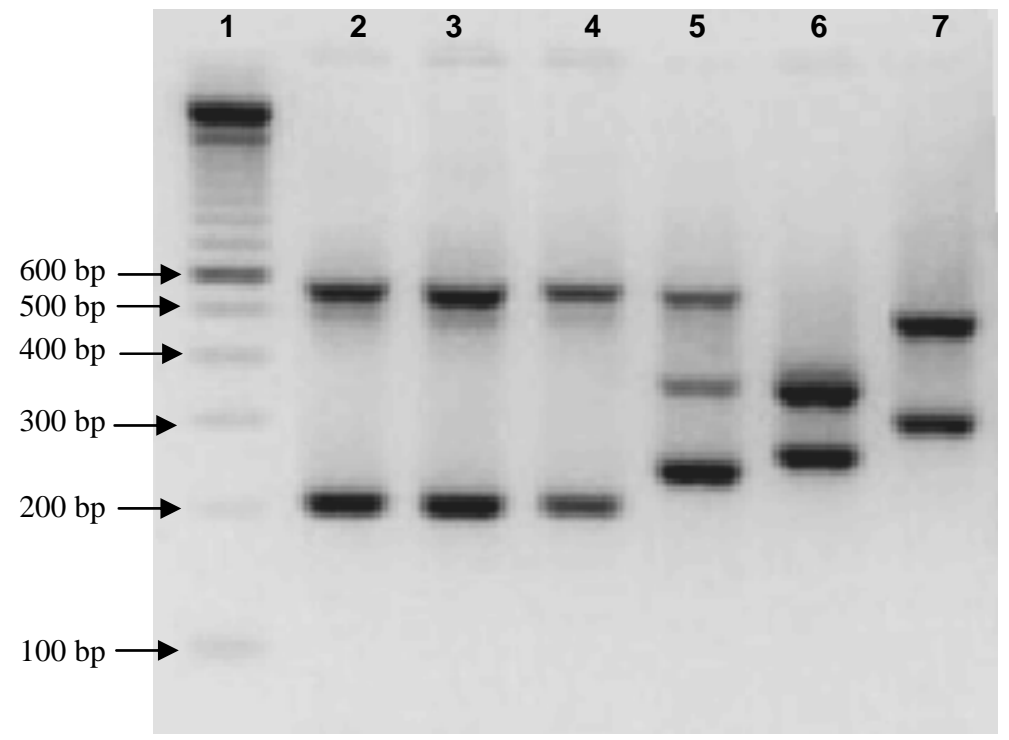

Fig. 5. VNTR pattern of ciprofloxacin resistant $S$. typhi isolates with that of comparative sensitive isolates (Lane 1: $100 \mathrm{bp}$ marker; Lane 2-4: Ciprofloxacin resistant isolates (MIC $1024 \mu \mathrm{g} / \mathrm{ml}$ ), Lane 5-7: Ciprofloxacin sensitive isolates, MIC 0.032, 0.064, $0.25 \mu \mathrm{g} / \mathrm{ml}$, respectively).

When looking for reasons for the rapidly increased quinolone resistance in Salmonella typhi isolates, three issues must be considered: transferable resistance, mutational resistance, and clonal spread. Until now, transferable resistance to the quinolone 
antimicrobial group has been described in one preliminary report. ${ }^{(5)}$ As far as we know, however, transferable fluoroquinolone resistance appears to be rare in bacteria in vivo. Thus, either clonal spread or resistance due to mutations in chromosomal genes remains the potential mechanism accounting for the high level of reduced fluoroquinolone susceptibility to fluoroquinolone in Bangladesh as well as in Southeast Asia. In Bangladesh, the possibility of clonal spread as a major contributing factor was excluded by identification of 8 serotypes among the quinolone-resistant isolates. In addition, some of these serotypes contained different antimicrobial resistance patterns along with their different VNTR patterns. Based on these data, we can conclude that the reduced fluoroquinolone susceptibility of Salmonella typhi in Bangladesh primarily involves mutations in the chromosomal genes. This concept is consistent with our VNTR experiment and finally proved by sequencing data where all 8 Salmonella isolates with reduced fluoroquinolone susceptibility were analyzed in ICDDR,B have shown point mutation leading to nucleotides change in their QRDR of the gyrase $A$ gene.

Taq-DNA polymerase successfully amplified the 195 bps gyrase $A$ gene from the genomic DNA of ciprofloxacin sensitive and ciprofloxacin resistant Salmonella serover typhi using primer of gyraseA (Fig. 6)

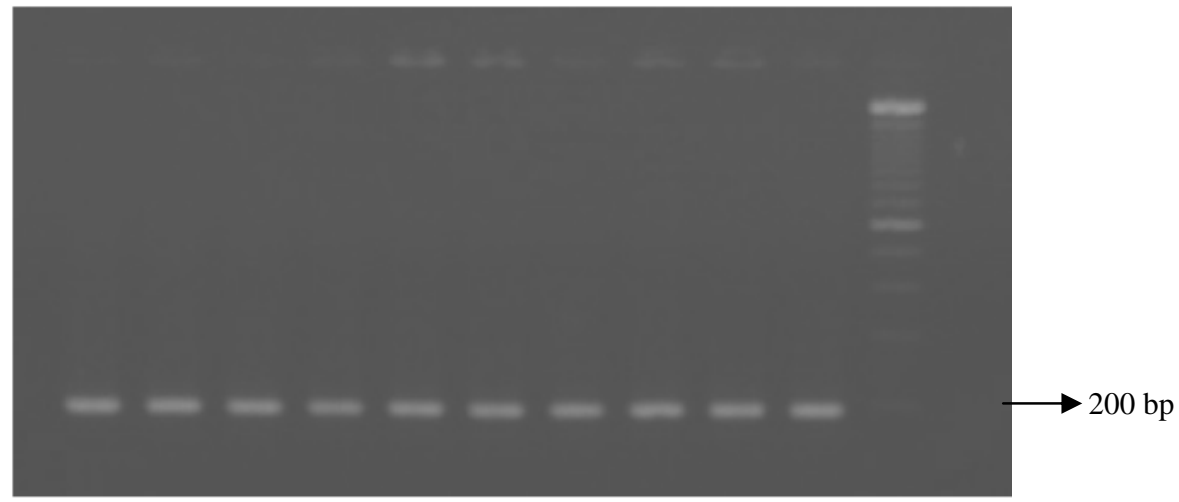

Fig. 6. Amplified gyraseA gene using PCR.

These PCR amplified products then showed different multiple fragments upon the treatment of Hinf-1 which showed a clarifying image indicating point mutation at different loci of QRDR region of the gyraseA gene (Fig. 7).

It is noteworthy that the less susceptible subpopulation has already undergone one point mutation and thus is potentially inclined to a second mutation, which leads to high level fluoroquinolone resistance. Admittedly, fluoroquinolone-resistant Salmonella strains are still extremely rare, and they are usually counter selected in field conditions.(6) Even so, one can think of highly fluoroquinolone-resistant Salmonella strains will inevitably emerge, if the less susceptible strains and quinolone pressure coexist. On this account, 
global surveillance on reduced fluoroquinolone susceptibility of Salmonella also is cared for epidemiologic reasons.

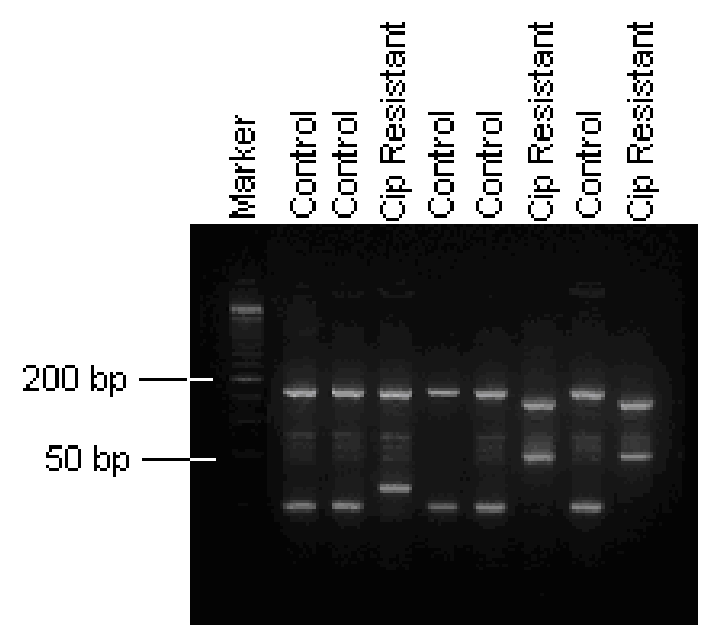

Fig. 7. Hinf-1 treated fragments of gyrase- $A$ PCR products.

The orchestrate increase in the incidence of fluoroquinolone resistance in Salmonella and other enteric bacteria, especially Campylobacter sp. and E. coli( $(7-10)$, drives a situation that impede the effectiveness of this antimicrobial group. The significantly common multi drug resistance observed here among the fluoroquinolone-resistant Salmonella compared with the susceptible population $(47.4 \%$ vs. $11.5 \%)$ is also a matter of concern. This finding suggests that the abrupt use of fluoroquinolone for multi drug resistant Salmonella provoke a question of whether the same could happen to other bacterial species. In that case, the likelihood of the emergence of notable pathogens resistant to fluoroquinolones as well as to other commonly used drugs will certainly increase if the consumption of fluoroquinolones continues to grow. Collectively, these data indicate that prudent use of the fluoroquinolone antimicrobial group is warranted to prevent further development of resistance and to preserve the usefulness of this valuable drug.

In conclusion, it has been shown that reduced susceptibility of Salmonella to the fluoroquinolone group was significantly associated with multi drug resistance. Moreover, all quinolone-resistant Salmonella isolates had undergone a point mutation in the QRDR of the gyrA gene. In contrast to previous reports on quinolone resistance in a specific clone or in a few Salmonella serotypes, the reduced fluoroquinolone susceptibility of our isolates was nonclonal. These data provide further evidence of the rapid spread of multidrug-resistant pathogens from one continent to another. The emergence of antimicrobial resistant pathogen in any part of the world may have global implication and is therefore a universal concern. 


\section{References}

1. Levine MM 1998. Typhoid fever. In: Evans AS, Brachman PS, (eds). Bacterial Infections of Human. Epidemiology and Control. Third edn. New York, Plenum Medical Book Company, pp. 839-58.

2. Reyna F, M Huesca, V Gonzalez and L Y Fuchs 1995. Salmonella typhimurium gyrA mutations associated with fluoroquinolone resistance. Antimicrob Agents Chemother 39: 16211623.

3. National Committee for Clinical Laboratory Standards. 1990. Methods for dilutional antimicrobial susceptibility tests for bacteria that grow aerobically. M7-A2. National Committee for Clinical Laboratory Standards, Villanova, Pennsylvania, USA.

4. National Committee for Clinical Laboratory Standards. Performance standards for antimicrobial disk susceptibility tests (Approved Standard M2-A5), 1993. National Committee for Clinical Laboratory Standard, Vallinova, Pennsylvania, USA.

5. Martínez-Martínez L, A Pascual, GA Jacoby 1998. Quinolone resistance from a transferable plasmid. Lancet 351: 797-799.

6. Giraud E, A Brisabois, JL Martel, E Chaslus-Dancla 1999. Comparative studies of mutations in animal isolates and experimental in vitro and in vivo selected mutants of Salmonella spp. suggest a counter selection of highly fluoroquinolone-resistant strains in the field. Antimicrob Agents Chemother 43: 2131-2137.

7. Smith KE, JM Besser, CW Hedberg, FT Leano, JB Bender, JH Wicklund 1999. Quinoloneresistant Campylobacter jejuni infections in Minnesota, 1992-1998. N. Engl. J. Med. 340: 1525-1532.

8. Sáenz Y, M Zarazaga, M Lantero, MJ Gastañares, F Baquero, C Torres 2000. Antibiotic resistance in Campylobacter strains isolated from animals, foods, and humans in Spain in 1997-1998. Antimicrob Agents Chemother 44: 267-271.

9. Prats G, B Mirelis, T Llovet, C Muñoz, E Miró, F Navarro 2000. Antibiotic resistance trends in enteropathogenic bacteria isolated in 1985-1987 and 1995-1998 in Barcelona. Antimicrob Agents Chemother 44: 1140-1145.

10. Garau J, M Xercavins, M Rodríguez-Carballeira, JR Gómez-Vera, I Coll, D Vidal 1999. Emergence and dissemination of quinolone-resistant Escherichia coli in the community. Antimicrob Agents Chemother 43: 2736-2741. 